\title{
Hybrid Control of a Truck and Trailer Vehicle*
}

\author{
Claudio Altafini ${ }^{1}$, Alberto Speranzon ${ }^{2 \star \star}$, and Karl Henrik Johansson ${ }^{2}$ \\ 1 SISSA-ISAS International School for Advanced Studies, \\ via Beirut 4, 34014 Trieste, Italy, \\ altafini@ma.sissa.it \\ 2 Department of Signals, Sensors and Systems, Royal Institute of Technology, \\ SE-10044 Stockholm, Sweden, \\ albspe@s3.kth.se, kallej@s3.kth.se
}

\begin{abstract}
A hybrid control scheme is proposed for the stabilization of backward driving along simple paths for a miniature vehicle composed of a truck and a two-axle trailer. When reversing, the truck and trailer can be modelled as an unstable nonlinear system with state and input saturations. Due to these constraints the system is impossible to globally stabilize with standard smooth control techniques, since some initial states necessarily lead to that the so called jack-knife locks between the truck and the trailer. The proposed hybrid control method, which combines backward and forward motions, provide a global attractor to the desired reference trajectory. The scheme has been implemented and successfully evaluated on a radio-controlled vehicle. Results from experimental trials are reported.
\end{abstract}

\section{Introduction}

Control of kinematic vehicles is an intensive research area with problems such as trajectory tracking, motion planning, obstacle avoidance etc. For a recent survey see [6, 5, 16]. The current paper discusses the problem of automatically reversing the truck and trailer system shown in Figure 1 The miniaturized vehicle is a 1:16 scale of a commercial vehicle and reproduces in detail its geometry. The vehicle is radio-controlled, has four axles, an actuated front steering, and an actuated second axle. According to the theory of vehicle control, our system is a general 3-trailer, because of the kingpin hitching between the second axle and the dolly. The off-axle connection is important, since it indicates that the system is neither differentially flat [19] nor feedback linearizable [20]. Hence, motion planning techniques, like those based on algebraic tools [10. 25] cannot be applied. Like a full-scale truck and trailer, our vehicle presents saturations on the steering angle and on the two relative angles between the bodies. These constraints, which are often overlooked in the literature, are of major concern here. The control task is to drive the vehicle backward along a preassigned path.

\footnotetext{
* This work was supported by the Swedish Foundation for Strategic Research through its Center for Autonomous Systems at the Royal Institute of Technology.

** Corresponding author.
} 


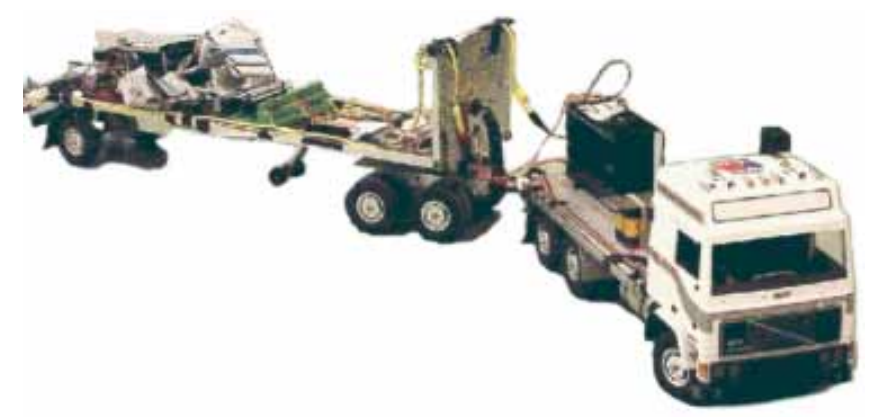

Fig. 1. Radio-controlled truck and trailer used in the experiments.

This problem is quite challenging, due to the unstable nonlinear dynamics and the state and input constraints.

The main contribution of the paper is a new hybrid feedback control scheme to stabilize the backward motion of the truck and trailer. It is argued that backward driving along a given line is impossible from a generic initial condition with a single controller. Instead we suggest a hybrid control strategy, where three different low-level controls are applied: one for backward driving along a line, one for backward driving along an arc of a circle, and one for forward driving. By switching between these control strategies, it is possible to solve the problem. The control design can be viewed as an exercise in hierarchical control design [26], where the control problem is divided into tasks which individually can be solved using standard control techniques.

A hybrid control scheme for stabilizing Dubins vehicle [9] is proposed in [3]. Backward steering control for other vehicle configurations are considered in $[8$, 13, 15] 17, 18, 23. For further discussion on the particular vehicle in this paper, see [2] 1]. The outline of the paper is as follows. The model of the system is presented in Section 2 In Section 3 the switching control scheme is presented together with the design of the low-level controls. Analysis of the switching controller is presented in Section 4. Experimental results are shown in Section 5.

\section{Modeling}

A nonlinear dynamic model for the truck and trailer vehicle is presented in this section. Linearized versions, which will be used in the control design, are given, and state constraints are discussed.

\subsection{Nonlinear Model}

A schematic picture of the truck and trailer system is shown in Figure 2 The system consists of three links indexed 1,2 , and 3 . Let $\left(x_{3}, y_{3}\right)$ be the cartesian 


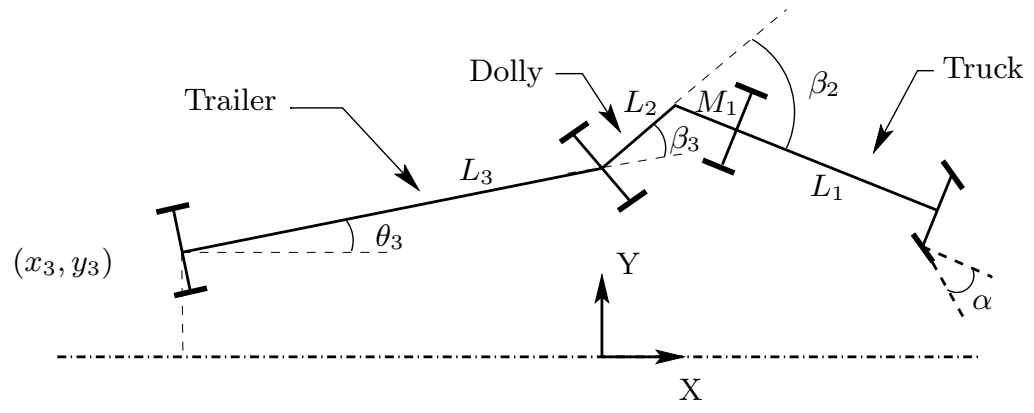

Fig. 2. Schematic picture of the system.

coordinates of the midpoint of the rearmost axle, $\theta_{3}$ the absolute orientation angle of that axle, $\beta_{2}$ the relative orientation angle between the dolly and the truck body, $\beta_{3}$ the relative orientation angle between the rearmost trailer body and the dolly, and $\alpha$ the steering angle. The lengths of the body parts are denoted $L_{1}, L_{2}, L_{3}$, and $M_{1}$, as indicated in the figure. For the miniature vehicle, we have $L_{1}=0.35 \mathrm{~m}, L_{2}=0.22 \mathrm{~m}, L_{3}=0.53 \mathrm{~m}$, and $M_{1}=0.12 \mathrm{~m}$. The kinematics are described by the following equations:

$$
\begin{aligned}
& \dot{x}_{3}=v \cos \beta_{3} \cos \beta_{2}\left(1+\frac{M_{1}}{L_{1}} \tan \beta_{2} \tan \alpha\right) \cos \theta_{3} \\
& \dot{y}_{3}=v \cos \beta_{3} \cos \beta_{2}\left(1+\frac{M_{1}}{L_{1}} \tan \beta_{2} \tan \alpha\right) \sin \theta_{3} \\
& \dot{\theta}_{3}=v \frac{\sin \beta_{3} \cos \beta_{2}}{L_{3}}\left(1+\frac{M_{1}}{L_{1}} \tan \beta_{2} \tan \alpha\right) \\
& \dot{\beta}_{3}=v \cos \beta_{2}\left(\frac{1}{L_{2}}\left(\tan \beta_{2}-\frac{M_{1}}{L_{1}} \tan \alpha\right)-\frac{\sin \beta_{3}}{L_{3}}\left(1+\frac{M_{1}}{L_{1}} \tan \beta_{2} \tan \alpha\right)\right) \\
& \dot{\beta}_{2}=v\left(\frac{\tan \alpha}{L_{1}}-\frac{\sin \beta_{2}}{L_{2}}+\frac{M_{1}}{L_{1} L_{2}} \cos \beta_{2} \tan \alpha\right)
\end{aligned}
$$

where the control inputs are the steering angle $(\alpha)$ and the longitudinal velocity at the second axle $(v)$. The sign of $v$ gives the direction of motion: $v>0$ corresponds to forward motion and $v<0$ to backward motion. All the variables are measurable using the sensors mounted on the system. We are interested in stabilizing the system along simple paths such as straight lines and arcs of circles. In most cases, the position variable $x_{3}$ will be neglected. Therefore, define the configuration state $\mathbf{p}=\left[y_{3}, \theta_{3}, \beta_{3}, \beta_{2}\right]^{T}$. The state equations can then be written as

$$
\dot{\mathbf{p}}=v(\mathcal{A}(\mathbf{p})+\mathcal{B}(\mathbf{p}, \alpha))
$$


Note that the drift is linear in the longitudinal velocity $v$. Since we are interested in stabilization around paths, we may introduce the arclength $d s \triangleq v d t$ and consider

$$
\frac{d \mathbf{p}}{d s}=\frac{v}{|v|}(\mathcal{A}(\mathbf{p})+\mathcal{B}(\mathbf{p}, \alpha))
$$

From this expression, we see that only the sign of $v$ matters. In the following, we therefore assume that $v$ takes value in the index set $\mathcal{I} \triangleq\{ \pm 1\}$.

\subsection{Linearization along Trajectories}

The steering angle $\alpha$ can be controlled such that the system (1) is asymptotically stabilized along a given trajectory. The stabilizing controller in each discrete mode of the hybrid controller will be based on LQ control. For the purpose of deriving these controllers, we linearize the system along straight lines and circular arcs.

Straight Line A straight line trajectory of (1) corresponds to an equilibrium point $(\mathbf{p}, \alpha)=\left(\mathbf{p}_{e}, \alpha_{e}\right)$ of (2) with $\mathbf{p}_{e}=\mathbf{0}$ and the steering input $\alpha_{e}=0$. Linearizing the system (2) around this equilibrium point yields

$$
\begin{aligned}
\dot{\mathbf{p}} & =v\left(\left(\left.\frac{\partial \mathcal{A}(\mathbf{p})}{\partial \mathbf{p}}\right|_{\left(\mathbf{p}_{e}\right)}+\left.\frac{\partial \mathcal{B}(\mathbf{p}, \alpha)}{\partial \mathbf{p}}\right|_{\left(\mathbf{p}_{e}, \alpha_{e}\right)}\right)\left(\mathbf{p}-\mathbf{p}_{e}\right)+\left.\frac{\partial \mathcal{B}(\mathbf{p}, \alpha)}{\partial \alpha}\right|_{\left(\mathbf{p}_{e}, \alpha_{e}\right)}\left(\alpha-\alpha_{e}\right)\right) \\
& =v(A \mathbf{p}+B \alpha)
\end{aligned}
$$

where

$$
A=\left.\frac{\partial \mathcal{A}(\mathbf{p})}{\partial \mathbf{p}}\right|_{(0)}=\left[\begin{array}{cccc}
0 & 1 & 0 & 0 \\
0 & 0 & 1 / L_{3} & 0 \\
0 & 0 & -1 / L_{3} & 1 / L_{2} \\
0 & 0 & 0 & -1 / L_{2}
\end{array}\right], \quad B=\left.\frac{\partial \mathcal{B}(\mathbf{p}, \alpha)}{\partial \mathbf{p}}\right|_{(0,0)}=\left[\begin{array}{c}
0 \\
0 \\
-M_{1} /\left(L_{1} L_{2}\right) \\
\left(L_{2}+M_{1}\right) /\left(L_{1} L_{2}\right)
\end{array}\right]
$$

The characteristic polynomial is

$$
\operatorname{det}(s I-v A)=s^{2}\left(s+\frac{v}{L_{2}}\right)\left(s+\frac{v}{L_{3}}\right)
$$

Hence the system is stable in forward motion $(v>0)$, but unstable in backward motion $(v<0)$. The presence of kingpin hitching (i.e. $M_{1} \neq 0$ ) makes the system not differentially flat (see [19]) and not feedback equivalent to chained form. What this implies can be seen considering the linearization (3) and the transfer function from $\alpha$ to $y_{3}$ :

$$
C(s I-v A)^{-1} v B=v^{3} M_{1} \frac{\left(v / M_{1}-s\right)}{L_{1} L_{2} L_{3} \operatorname{det}(s I-v A)}
$$

The presence of the kingpin hitching introduces zero dynamics in the system. The zero dynamics is unstable if $v>0$ and stable otherwise. When $M_{1}=0$ the system can be transformed into a chain of integrators by applying suitable feedback [21, 24]. 
Circular Arc Consider the subsystem of (II) corresponding to the state $\overline{\mathbf{p}}=$ $\left[\beta_{3}, \beta_{2}\right]^{T}$ and denote it as

$$
\dot{\overline{\mathbf{p}}}=v(\overline{\mathcal{A}}(\overline{\mathbf{p}})+\overline{\mathcal{B}}(\overline{\mathbf{p}}, \alpha))
$$

A circular arc trajectory of (10) is then an equilibrium point $\left(\overline{\mathbf{p}}_{e}, \alpha_{e}\right)$ of (7), with $\alpha_{e}$ being a fixed steering angle and $\overline{\mathbf{p}}_{e}=\left[\beta_{3 e}, \beta_{2 e}\right]^{T}$ being given by

$$
\beta_{2 e}=\arctan \left(\frac{M_{1}}{r_{1}}\right)+\arctan \left(\frac{L_{2}}{r_{2}}\right), \quad \beta_{3 e}=\arctan \left(\frac{r_{3}}{L_{3}}\right)
$$

where $r_{1}=L_{1} / \tan \alpha_{e}, r_{2}=\sqrt{r_{1}^{2}+M_{1}^{2}-L_{2}^{2}}$, and $r_{3}=\sqrt{r_{2}^{2}-L_{3}^{2}}$ are the radii of the circular trajectories of the three rear axles. Linearization of (7) around $\left(\overline{\mathbf{p}}_{e}, \alpha_{e}\right)$ gives

$$
\dot{\overline{\mathbf{p}}}=v\left(\bar{A}\left(\overline{\mathbf{p}}-\overline{\mathbf{p}}_{e}\right)+\bar{B}\left(\alpha-\alpha_{e}\right)\right)
$$

where

$\bar{A}=\left[\begin{array}{cc}\frac{\cos \beta_{2 e} \cos \beta_{3 e}}{L_{3}} \frac{\cos \beta_{2 e}}{L_{2}}+\frac{\sin \beta_{2 e} \sin \beta_{3 e}}{L_{3}}+\frac{M_{1}}{L_{1}}\left(\frac{\sin \beta_{2 e}}{L_{2}}-\frac{\cos \beta_{2 e} \sin \beta_{3 e}}{L_{3}}\right) \tan \alpha_{e} \\ 0 & -\frac{\cos \beta_{2 e}}{L_{2}}\left(1+\frac{M_{1}}{L_{1}} \tan \beta_{2 e} \tan \alpha_{e}\right)\end{array}\right]$ $\bar{B}=\left[\begin{array}{c}-\frac{M_{1}}{L_{1}}\left(\frac{\cos \beta_{2 e}}{L_{2}}+\frac{\sin \beta_{2 e} \sin \beta_{3 e}}{L_{3}}\right)\left(1+\tan ^{2} \alpha_{e}\right) \\ \frac{1}{L_{1}}\left(1+\frac{M_{1}}{L_{2}} \cos \beta_{2 e}\right)\left(1+\tan ^{2} \alpha_{e}\right)\end{array}\right]$

\subsection{State and Input Constraints}

An important feature of the truck and trailer vehicle is its input and the state constraints. In particular, for the considered miniature vehicle we have the following limit for the steering angle

$$
|\alpha| \leq \alpha_{s}=0.43 \mathrm{rad}
$$

and for the relative angles

$$
\left|\beta_{2}\right| \leq \beta_{2 s}=0.6 \mathrm{rad}, \quad\left|\beta_{3}\right| \leq \beta_{3 s}=1.3 \mathrm{rad}
$$

A consequence of the latter two constraints is the appearing of the so called jackknife configurations, which correspond to at least one of the relative angles $\beta_{2}$ and $\beta_{3}$ reaching its saturation value. When the truck and trailer is this configuration, it is not able to push anymore the trailer backwards. The states $y_{3}$ and $\theta_{3}$ do not present saturations. Due to limited space when maneuvering, however, it is convenient to impose the constraints

$$
\left|y_{3}\right| \leq y_{3 s}=0.75 \mathrm{~m}, \quad\left|\theta_{3}\right| \leq \theta_{3 s}=\pi / 2 \mathrm{rad}
$$




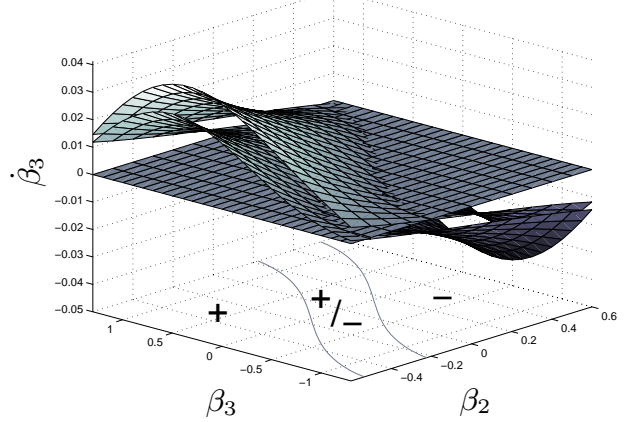

Fig. 3. The right-hand side of equation (1d) as a function of $\beta_{2}$ and $\beta_{3}$ for $\alpha= \pm \alpha_{s}$. For certain choices of $\left(\beta_{2}, \beta_{3}\right)$, the input constraint on $\alpha$ leads to the jack-knife configuration since for these values both $\dot{\beta}_{3}$ and $\beta_{3}$ are positive. The plus and minus signs indicate the $\left(\beta_{2}, \beta_{3}\right)$ regions where $\dot{\beta}_{3}$ is necessarily positive and negative, respectively, regardless of $\alpha$.

The domain of definition of $\mathbf{p}$ is thus given by

$$
D=\left(-y_{3 s}, y_{3 s}\right) \times\left(-\theta_{3 s}, \theta_{3 s}\right) \times\left(-\beta_{3 s}, \beta_{3 s}\right) \times\left(-\beta_{2 s}, \beta_{2 s}\right)
$$

Note that the since the steering driver of the miniature vehicle tolerates very quick variations, we do not assume any slew rate limitations on $\alpha$.

\section{Switching Control}

The switched control strategy is presented in this section together with the lowlevel controls, but first some motivation for investigating switching controls are discussed.

\subsection{Why Switching Control?}

It is easy to show that due to the saturations of the input and the state, it is not possible to globally stabilize the truck and trailer along a straight line using only backward motion. Consider the right-hand side of equation (1d) for $v=-1$, and note that $\dot{\beta}_{3}$ depends on $\beta_{2}, \beta_{3}$, and $\alpha$. The two surfaces in Figure 3 show how $\dot{\beta}_{3}$ depends on $\beta_{2}$ and $\beta_{3}$ for the two extreme cases of the steering angle $\alpha$, i.e., $\alpha=-\alpha_{s}$ and $\alpha=\alpha_{s}$, respectively. It follows that there are initial states such that both $\beta_{3}$ and $\dot{\beta}_{3}$ are positive, regardless of the choice of $\alpha$ (for example, $\beta_{2}=-\beta_{2 s}$ and $\beta_{3}=\beta_{3 s}$ ). Starting in such a state leads necessarily to that the truck and trailer vehicle ends up in the jack-knife configuration, when driving backwards. Naturally, this leads to the idea of switching the control between backward and forward motion (as a manual driver would do). Before 

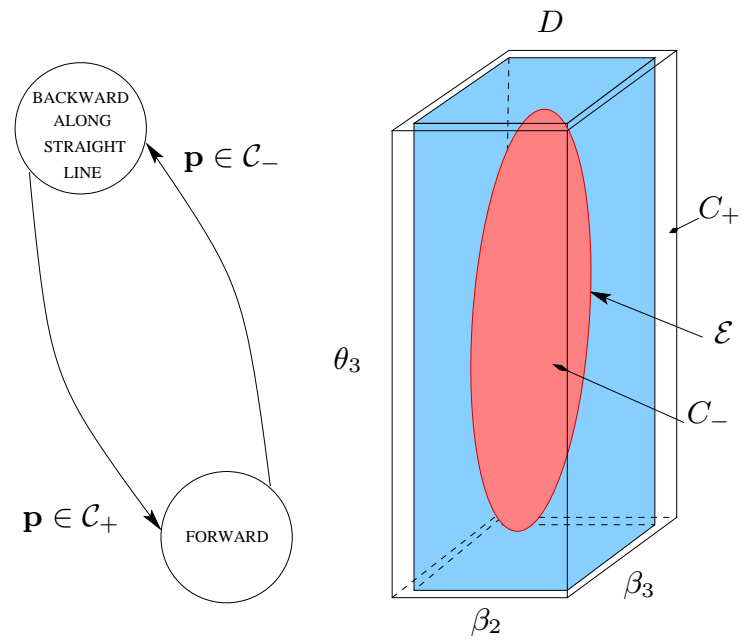

Fig. 4. Two states switching control and a picture of the sets $\mathcal{C}_{-}$and $\mathcal{C}_{+}$with respect to $\mathcal{E}$.

we present how this switching can be done, note that even without input and state constraints it is not possible to use the same state feedback controller in forward and backward motion. This follows simply from that if the system (2) with $v=1$ is asymptotically stable for a smooth control law $\alpha=-K(\mathbf{p})$, then the corresponding system with $v=-1$ is unstable.

\subsection{Switching Control Strategy}

A simplified version of the proposed hybrid control is shown to the left in Figure 4. The hybrid automaton consists of two discrete modes: backward driving along a straight line and forward driving. The switchings between the modes occur when the continuous state reach certain manifolds. The control design consists now of two steps: choosing these manifolds and determining local controllers that stabilize the system in each discrete mode. Suppose a stabilizing control law $\alpha=-K_{B}(\mathbf{p})$ has been derived for the backward motion $(v=-1)$ of the system (2). Let $\mathcal{E} \subset D$ denote the largest ellipsoid contained in the region of attraction for the closed-loop system. From the discussion in previous section, we know that $\mathcal{E}$ is not the whole space $D$. If the initial state $\mathbf{p}(0) \in D$ is outside $\mathcal{E}$, then $K_{B}$ will not drive the state to the origin. As proposed by the hybrid controller in Figure 4, we switch in that case to forward mode $(v=1)$ and the control law $\alpha=-K_{F}(\mathbf{p})$. The forward control $K_{F}$ is chosen such that the trajectory is driven into $\mathcal{E}$. When the trajectory reaches $\mathcal{E}$, we switch to backward motion. The sets $\mathcal{C}_{-} \subset D$ and $\mathcal{C}_{+} \subset D$ on the edges of the hybrid automaton in Figure 4 define the switching surfaces. To avoid chattering due to measurement noise and to add robustness to the scheme, the switching does not take place 
exactly on the surface of $\mathcal{E}$. Instead $\mathcal{C}_{-}$is slightly smaller than $\mathcal{E}$, and $\mathcal{C}_{+}$is larger than $\mathcal{E}$, see the sketch to the right in Figure 4, It is reasonable to choose $\mathcal{C}_{-}$(the set defining the switch from forward to backward mode) of the same shape as $\mathcal{E}$, but scaled with a factor $\rho \in(0,1)$. There is a trade-off in choosing $\rho$ : if $\rho$ is close to one, then the system will be sensitive to disturbances; and if $\rho$ is small, then the convergence will be slow since the forward motion will be very long. In the implementations we chose $\rho$ in the interval $(0.7,0.8)$. The set $\mathcal{C}_{+}$(defining the switch from backward to forward mode) is chosen as a rescaling of $D$. In the implementation, the factors were selected as unity in the $y_{3}$ and the $\theta_{3}$ component, but 0.8 and 0.7 in the $\beta_{2}$ and $\beta_{3}$ component, respectively. The choice is rather arbitrary. The critical point is that $\beta_{2}$ and $\beta_{3}$ should not get too close to the jack-knife configuration $\left(\left|\beta_{2}\right|=\beta_{2 s}\right.$ and $\left.\left|\beta_{3}\right|=\beta_{3 s}\right)$. Experiments on the miniature vehicle with the hybrid controller in Figure 4 implemented show that time spent in the forward mode is unacceptably long. The reason is that the time constant of $\theta_{3}$ is large. To speed up convergence, we introduce an intermediate discrete mode which forces $\theta_{3}$ to recover faster. This alignment control mode corresponds, for example, to reversing along an arc of circle. The complete switching controller is shown in Figure 5. Thus, the hybrid automaton consists of three discrete modes: backward driving along a straight line, backward driving along an arc of a circle, and forward driving. The switchings between the discrete modes are defined by the following sets:

$$
\begin{aligned}
& \Omega=\left\{\mathbf{p}=\left[y_{3}, \theta_{3}, \beta_{3}, \beta_{2}\right]^{T} \in D:\left|\theta_{3}\right|<\tilde{\theta}_{3} \text { or } y_{3} \theta_{3}<0\right\} \\
& \Psi=\left\{\mathbf{p}=\left[y_{3}, \theta_{3}, \beta_{3}, \beta_{2}\right]^{T} \in D:\left|\theta_{3}\right|<\tilde{\theta}_{3} / 2,\left|y_{3}\right|<\tilde{y}_{3}\right\} \\
& \Phi=\left\{\mathbf{p}=\left[y_{3}, \theta_{3}, \beta_{3}, \beta_{2}\right]^{T} \in D:\left[0,0, \beta_{3}, \beta_{2}\right]^{T} \in \mathcal{C}-\right\}
\end{aligned}
$$

where $\tilde{\theta}_{3}$ and $\tilde{y}_{3}$ are positive design parameters. In the implementation we choose $\tilde{\theta}_{3}=0.70 \mathrm{rad}$ and $\tilde{y}_{3}=0.02 \mathrm{~m}$. In the figure, recall that $\Omega^{c}$ denotes the complement of $\Omega$. The interpretation of the switching conditions in Figure 5 are as follows. Suppose the initial state $\mathbf{p}(0)$ is in $\mathcal{C}_{+}$(thus outside the region of attraction for the backward motion system) and that the hybrid controller starts in the forward mode. The system stays in this mode until $\beta_{2}$ and $\beta_{3}$ are small enough, i.e., until $\left(\beta_{2}, \beta_{3}\right)$ belongs to the ellipse defined by $\Phi$. Then a switch to the alignment control mode for backward motion along an arc of a circle occurs. The system stays in this mode until $\left|y_{3}\right|$ is sufficiently small, when a switch is taken to the mode for backward motion along a straight line. The other discrete transitions in Figure 5 may be taken either due to that the alignment originally is good enough or due to disturbances or measurement noise.

\subsection{Low-Level Controls}

In this section we briefly describe how the three individual state-feedback controllers $\alpha=-K(\mathbf{p})$, applied in each of the discrete modes of the hybrid controller, were derived and what heuristics that had to be incorporated. 


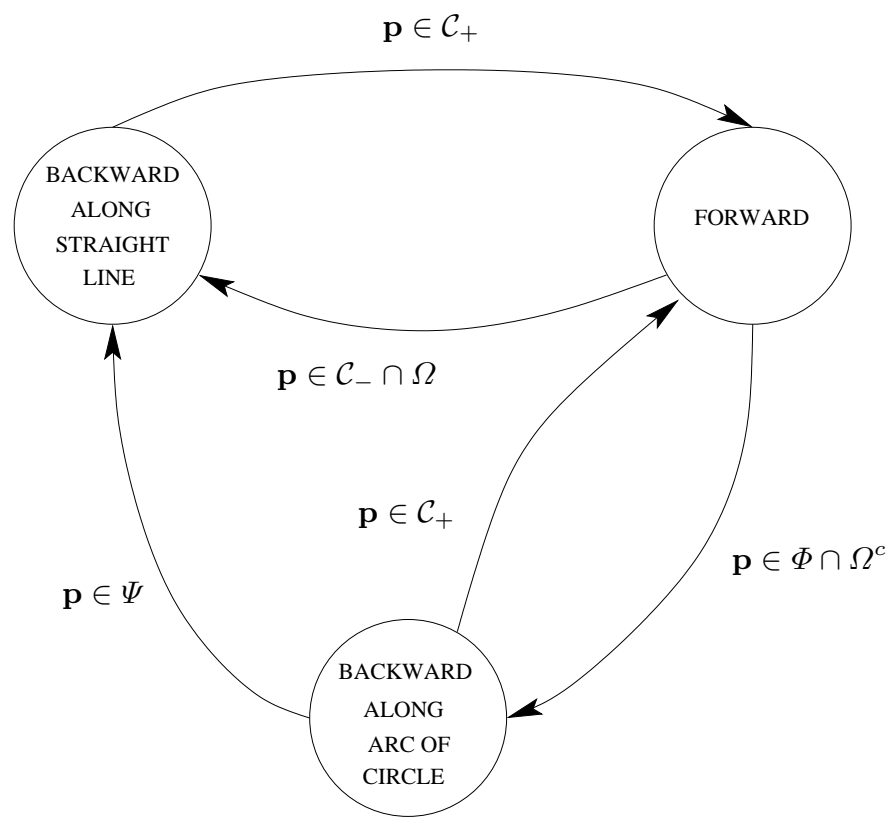

Fig. 5. Three states switching control.

Backward along Straight Line For the discrete mode for the backward motion along a straight line, we design a LQ controller $\alpha=-K_{B} \mathbf{p}$ based on the linearized model (3) with $v=-1$. The choice of cost criterion is

$$
J_{B}=\int_{0}^{\infty}\left(\mathbf{p}^{T} Q_{B} \mathbf{p}+\alpha^{2}\right) d t \quad Q_{B}=Q_{B}^{T}>0
$$

The heuristic we have adopted let us choose $Q_{B}$ as a diagonal matrix, where the $y_{3}$ weight is the smallest, the $\theta_{3}$ weight one order of magnitude larger, and the $\beta_{3}$ and $\beta_{2}$ weights another two orders of magnitude larger. The reason for having large weights on $\beta_{3}$ and $\beta_{2}$ is to avoid saturations. In general, the intuition behind this way of assigning weights reflects the desire of having decreasing closed-loop bandwidths when moving from the inner loop to the outer one. For example, the relative displacement $y_{3}$ is related to $\beta_{3}$ and $\beta_{2}$ through a cascade of two integrators, as can be seen from the linearization (4). It turns out that such a heuristic reasoning is very important in the practical implementation in order to avoid saturations.

Forward The state-feedback control in the forward mode is designed based on pole placement. Since closed-loop time constant of $y_{3}$ is of several orders of magnitude larger than for the other three states of $\mathbf{p}$, the measurement $y_{3}$ is not used in the forward controller. Instead consider the state $\hat{\mathbf{p}}=\left[\theta_{3}, \beta_{3}, \beta_{2}\right]^{T}$ and 
the corresponding linearized system. We choose a controller gain $K_{F}$ such that the linearized system has three closed-loop poles of the same order of magnitude.

Backward along Arc of Circle For the backward motion mode, we consider the stabilization of the relative angles $\overline{\mathbf{p}}=\left[\beta_{3}, \beta_{2}\right]^{T}$ of the corresponding linearized subsystem (9). The state-feedback controller $\alpha=-K_{A} \overline{\mathbf{p}}$ is derived based on LQ control. Recall that stabilizing the origin for (9) corresponds to stabilizing the truck and trailer along a circular trajectory.

\section{Analysis of Switching Control}

In this section, the closed-loop system with the switching controller is analyzed. First, a discussion on how to estimate the region of attraction for the reversing truck and trailer is presented, then a result on asymptotic stability for the hybrid control system is reviewed.

\subsection{Region of Attraction}

The switching conditions in the hybrid control scheme discussed in previous section were partially based on an estimate $\mathcal{E}$ of the region of attraction for the closed-loop system in backward motion. It is in general difficult to obtain an accurate approximation for the region of attraction, particularly for systems with state and input constraints [11. In this paper we rely on the numerical simulation of the closed-loop behavior. Hence, considering the nonlinear system (2) with $v=-1$ and closed-loop control $\alpha=-K_{B} \mathbf{p}$ :

$$
\dot{\mathbf{p}}=-\left(\mathcal{A}(\mathbf{p})+\mathcal{B}\left(\mathbf{p},-K_{B} \mathbf{p}\right)\right)
$$

In order to obtain a graphical representation of the results, we disregard $y_{3}$. This is reasonable as long as the initial condition $y_{3}(0)$ satisfies the artificial constraint $y_{3}(0) \leq y_{3 s}$ introduced in Section 2 Note that this constraint does not influence the analysis of the other states, since $y_{3}$ does not enter the differential equations (1c)-(1e). The black region in Figure 6 shows states $\hat{\mathbf{p}}=\left[\theta_{3}, \beta_{3}, \beta_{2}\right]^{T}$ that belong to the region of attraction. We notice that this cloud of initial conditions closely resembles an ellipsoid. The figure also shows an ellipsoid strictly contained in the region of attraction, which has simply been fitted by hand. Note that the considered problem is related to finding the reachability set for a hybrid system with nonlinear continuous dynamics. For our purposes, we used numerical simulations validated by practical experiments, in order to have a mathematical description of $\mathcal{E}$. It would be interesting to apply recent reachability tools [7, 4, 12, 14] on this highly nonlinear problem.

\subsection{Stability Analysis}

Consider system (2)

$$
\dot{\mathbf{p}}=v(\mathcal{A}(\mathbf{p})+\mathcal{B}(\mathbf{p}, \alpha))
$$




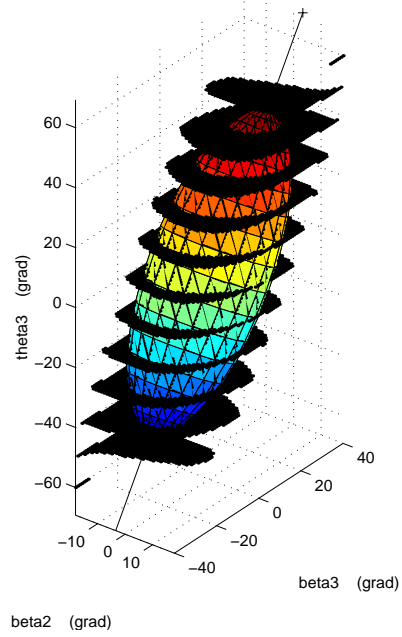

Fig. 6. Region of attraction (in black) for the backward motion obtained through simulation of the nonlinear closed-loop system. An approximating ellipsoid is fitted inside.

under the switching control defined in Figure 4. It is straightforward to show that there exists a stabilizing controller if full state-feedback is applied in both the backward and the forward mode, see [2. Note, however, that the low-level control for the forward motion discussed in previous section did not use feedback from $y_{3}$. Hence, we need a result on the boundedness of $y_{3}$. Such a bound can be derived and then under rather mild assumptions it can be proved that the closed-loop system with the two-state hybrid controller is asymptotically stable in a region only slightly smaller than $D$ (see [2] for details).

\section{Implementation and Experimental Results}

The controller for the truck and trailer shown in Figure 1 was implemented using a commercial version of PC/104 with an AMD586 processor and with an acquisition board for the sensor readings. The signals from the potentiometers for the relative angles $\beta_{2}$ and $\beta_{3}$ were measured via the $\mathrm{AD}$ converter provided with the acquisition board, while the position of the trailer was measured using two encoders, placed on the wheels of the rearmost axle. The sampling frequency was about $10 \mathrm{~Hz}$, which was sufficient since the velocity was very low. Figures 7 and 8 show an experiment that starts with a forward motion for the realignment of the trailer and truck, followed by a backward motion along an arc of circle. (The backward motion along a line is not shown, since the truck reached the wall before ending the manoeuvre). The entire motion of the system is depicted in the left of Figure [7] with the configurations at two instances for the forward 

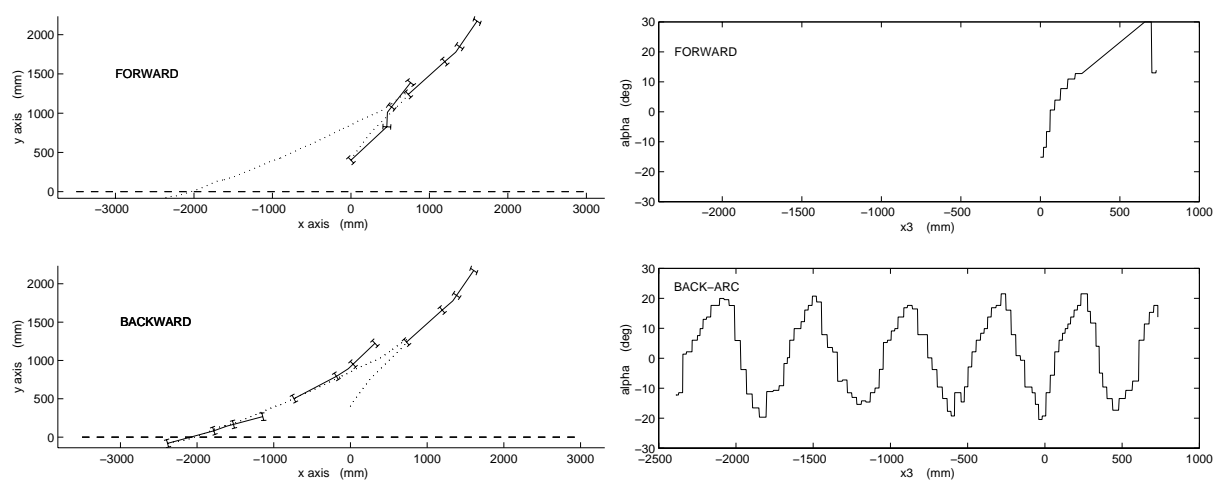

(a) Some positions of the system along the trajectory

(b) Input signal

Fig. 7. Experiment: sketch of the motion of the vehicle. Notice that the all the measures along $x_{3}$ are respect the center of the last axle of the trailer. The input signal is divided in two subplots one for the forward and the other backward (along an arc) motion.

motion and three for the backward. The input signal is shown in the right side of Figure 7 . The left graphs of Figure 8 show the state variable $y_{3}$ and $\theta_{3}$ relative to the manoeuvre of Figure [7] but with another scaling. The initial condition is $\beta_{2}(0)=-40 \mathrm{deg}$ and $\beta_{3}(0)=40 \mathrm{deg}$ with $\theta_{3}(0)=42 \mathrm{deg}$. This means that the initial condition is outside the ellipsoid $\mathcal{E}$, hence the hybrid controller starts in the forward motion mode. After a while, the two relative angles $\beta_{3}$ and $\beta_{2}$ are small (and thus the truck and trailer is realigned). This is illustrated to the right in Figure [8] which shows $\beta_{3}$ and $\beta_{2}$ as a function of $x_{3}$. Since $y_{3} \cdot \theta_{3}>0$, the controller now switches to the mode for backward along an arc of a circle. In total the system travels a distance of $2.5 \mathrm{~m}$ in the backward mode and $0.7 \mathrm{~m}$ in the forward mode. Some videos showing the motion of the system can be downloaded from 22 .

\section{References}

[1] C. Altafini. Controllability and singularities in the $n$-trailer system with kingpin hitching. In Proceedings of the 14th IFAC World Congress, volume Q, pages 139-145, Beijing, China, 1999.

[2] C. Altafini, A. Speranzon, and B. Wahlberg. A feedback control scheme for reversing a truck and trailer vehicle. Accepted for publication in IEEE Transaction on Robotics and Automation, 2001. 

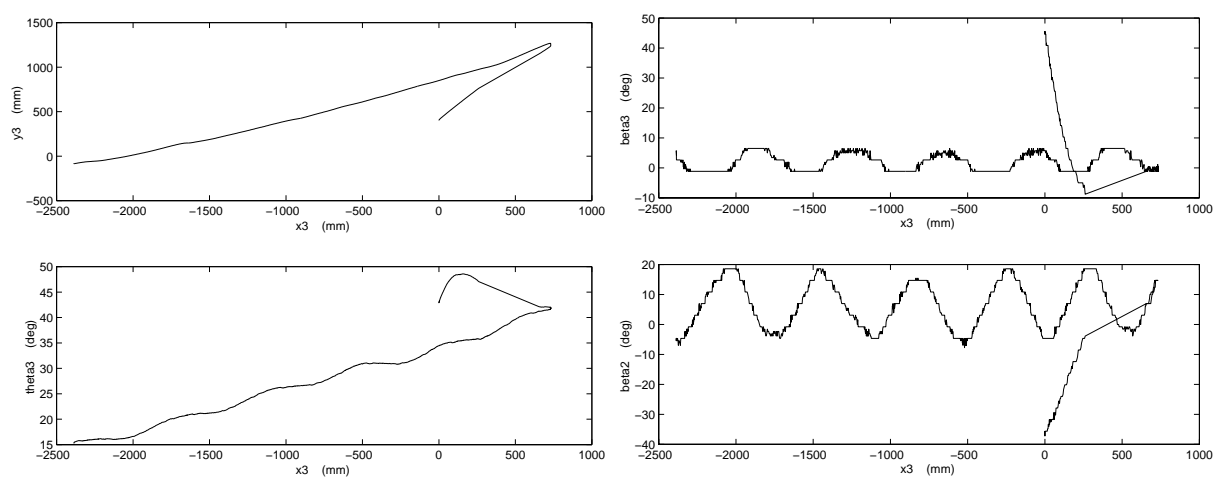

(a) State variables $y_{3}$ and $\theta_{3}$

(b) State variables $\beta_{3}$ and $\beta_{2}$

Fig. 8. Experiment: state variables $\left[y_{3}, \theta_{3}, \beta_{3}, \beta_{2}\right]$ relative to the maneuver shown in the previous picture.

[3] A. Balluchi, P. Soueres, and A. Bicchi. Hybrid feedback control for path tracking by a bounded-curvature vehicle. In Proceedings of the 4 th International Workshop, Hybrid System: Computation and Control, pages 133-146, Rome, Italy, 2001.

[4] O. Bournez, O. Maler, and A. Pnueli. Orthogonal polyhedra: Representation and computation. In F. Vaandrager and J. van Schuppen, editors, Hybrid systems: Computation and Control, volume 1569 of LNCS, 1999.

[5] C. Canudas de Wit. Trends in mobile robot and vehicle control. Lecture notes in control and information sciences. Springer-Verlag, 1998. In K.P. Valavanis and B. Siciliano (eds), Control problems in robotics.

[6] C. Canudas de Wit, B. Siciliano, and B. Bastin. Theory of robot control. SpringerVerlag, 1997.

[7] T. Dang and O. Maler. Reachability analysis via face lifting. In T.A. Henzinger and S. Sastry, editors, Hybrid Systems: Computation and Control, volume 1006 of LNCS, pages 96-109. Springer, 1998.

[8] A.W. Divelbiss and J. Wen. Nonholonomic path planning with inequality constraints. In Proceedings of IEEE Int. Conf. on Robotics and Automation, pages 52-57, 1994.

[9] L.E. Dubins. On curves of minimal length with a constraint on average curvature an with prescribed initial and terminal position and tangents. American Journal of Mathematics, 79:497-516, 1957.

[10] M. Fliess, J. Levine, P. Martin, and P. Rouchon. Flatness and defect of nonlinear systems: introductory theory and examples. Int. Journal of Control, 61(6):13271361, 1995.

[11] K.T. Gilbert, E.G.and Tan. Linear systems with state and control constraints: the theory and application of maximal output admissible set. IEEE Transactions on Automatic Control, 36:1008-1020, 1991.

[12] M.R. Greenstreet and M. Mitchell. Reachability analysis using polygonal projection. In In [VS99], pages 76-90, 1999. 
[13] D.H. Kim and J.H. Oh. Experiments of backward tracking control for trailer systems. In Proceedings IEEE Int. Conf. on Robotics and Automation, pages 19-22, Detroit, MI, 1999.

[14] A.B. Kurzhanski and P. Varaiya. Ellipsoidal techniques for reachability analysis. In N. In Lynch and B. Krogh, editors, Hybrd Systems: Computation and Control, volume 1790 of Lecture Notes in Computer Sciences, pages 203-213. SpringerVerlag, 2000.

[15] F. Lamiraux and J.P. Laumond. A practical approach to feedback control for a mobile robot with trailer. In 3291-3296, editor, Proc. IEEE Int. Conf. on Robotics and Automation, Leuven, Belgium, 1998.

[16] J.P. Laumond. Robot Motion Planning and Control. Lecture notes in control and information sciences. Springer-Verlag, 1998.

[17] W. Li, T. Tsubouchi, and S. Yuta. On a manipulative difficulty of a mobile robot with multiple trailers for pushing and towing. In Proceedings IEEE Int. Conf. on Robotics and Automation, pages 13-18, Detroit, Mi, 1999.

[18] Y. Nakamura, H. Ezaki, Y. Tan, and W. Chung. Design of steering mechanism and control of nonholonomic trailer systems. In Proceeding of IEEE Int. Conf. on Robotics and Automation, pages 247-254, San Francisco, CA, 2000.

[19] P. Rouchon, M. Fliess, J. Levine, and P. Martin. Flatness, motion planning and trailer systems. In Proc. 32nd IEEE Conf. on Decision and Control, pages 27002075, San Antonio, Texas, 1993.

[20] M. Sampei, T. Tamura, T. Kobayashi, and N. Shibui. Arbitrary path tracking control of articulated vehicles using nonlinear control theory. IEEE Transaction on Control Systems Technology, 3:125-131, 1995.

[21] O.J. Sørdalen. Conversion of the kinematics of a car with $n$ trailers into chained form. In Proc. IEEE Int. Conf. on Robotics and Automation, pages 382-387, Atlanta, Georgia, 1993.

[22] A. Speranzon. Reversing a truck and trailer vehicle. http://www.s3.kth.se/ albspe/truck/.

[23] K. Tanaka, T. Taniguchi, and H.O. Wang. Trajectory control of an articulated vehicle with triple trailers. In Proc. IEEE International Conference on Control Applications, pages 1673-1678, 1999.

[24] D. Tilbury, R. Murray, and S. Sastry. Trajectory generation for the n-trailer problem using Goursat normal form. IEEE Trans. on Automatic Control, 40:802819, 1995.

[25] M. van Nieuwstadt and R. Murray. Real time trajectory generation for differentially flat systems. International Journal of Robust and Nonlinear Control, 8(38):995-1020, 1998.

[26] P. Varaiya. Smart cars on smart roads: Problems of control. IEEE Transactions on Automatic Control, 38(2):195-207, February 1993. 